\title{
Oncoplastic Techniques in Central Breast Cancer: Approaches and Outcomes
}

\author{
Ahmed Gamal El-Din Osman, MD, MRCS; Kamal Mamdouh, MD \\ Department of General Surgery, Ain Shams University, Egypt
}

Breast cancer is the most common cancer among women and is the second leading cause of cancer deaths. Central tumors represent $5-20 \%$ of breast cancers. Tumors localized in the central quadrant have always represented a challenge for the surgeon because of the critical aesthetical matters related to the nippleareola complex (NAC).

Patients and methods: In this study, we have 30 patients with central breast cancer. Patients were divided into two groups, each is formed of fifteen patients, the first group underwent Grissoti technique and the other inferior pedicle technique with wide local excision of NAC with safety margins.

Conclusion: It is ideal for women with medium to large breasts, having a Paget's disease or harboring central tumors to perform these oncoplastic techniques which are considered to be safe and satisfactory options for women with retro-areolar malignancy wishing to preserve their breasts.

Key words: Central breast tumors, oncoplastic techniques, Grissoti

\section{Introduction}

Breast cancer is the most common type of malignancy among females in Egypt and worldwide where it represents $38.8 \%$ of the total cancer incidence locally and $22.9 \%$ globally. In 2013 , the estimated number of cases in Egypt was 17,905 and was expected to be triple by $2050 .{ }^{1}$ Breast conserving surgery (BCS) and radiotherapy were proved to provide same local control and survival rate to modified radical mastectomy in early breast cancer. ${ }^{2}$ The aim of BCS is to preserve the breast aesthetic while adhering to the oncologic principles.

Oncoplastic breast surgery (OBS) is defined as the simultaneous application of lumpectomy and reconstructive techniques with wider excision without compromising oncologic principles and provides esthetic closure of the glandular defect by plastic techniques. ${ }^{3}$

Central breast tumors represent a challenge to the surgeon who must fulfill the fundamental goals of BCS, namely adequate margins and acceptable appearance. $^{4}$

The aim of our study was to evaluate two approaches for excision of central breast tumors; Grissoti's technique for small and medium breast size and inferior pedicle mammoplasty for large breast; advantages and disadvantages, oncologic and aesthetic results.

\section{Patients and methods}

This prospective study was conducted on 30 female patients diagnosed to have central breast cancer in Ain Shams University Hospitals between
January 2013 to March 2016.

Criteria for case selection included: Central and retroareolar primary breast cancer not more than $1.5 \mathrm{~cm}$ away from nipple areola complex (NAC) of $\mathrm{T}_{\text {is-2 }} \mathrm{N}_{0-1} \mathrm{M}_{0}$ (TNM classification), imaging studies confirming patho-anatomic features corresponding to clinical symptoms, histopathological diagnosis confirming the clinical features and manifestation. Exclusion criteria included: Multicenteric breast cancer, distant metastases, previously treated ipsilateral breast cancer, inflammatory breast cancer, and tumors more than $1.5 \mathrm{~cm}$ from the areola. Non-oncological exclusion criteria included small size breast cup (A).

Patients were divided into two groups according to breast size; small to medium size breast cup (B-C) performed Grissott's technique and larger breast cup ( $D$ and more) performed the inferior pedicle technique.

It is important to balance a woman's breast size with the rest of her figure, breast size was estimated by cup size which represents the difference between 2 measures the first is chest circumference just below the breast at the infra-mammary fold, the second is the bust circumference representing the circumference at the most projecting part of the breast usually at the level of the nipples. The following table represents breast cup size chart (Table 1).

The follow-up protocol consisted of weekly follow up for 4 weeks, followed by 3-month and then 6 -month clinical examinations. 
Table 1: Breast Cup size

\begin{tabular}{ccc}
\hline $\begin{array}{l}\text { Difference in } \\
\text { CM }\end{array}$ & Difference in inch & Cup size \\
\hline 2.5 & 1 & $\mathrm{~A}$ \\
5 & 2 & $\mathrm{~B}$ \\
7.5 & 3 & $\mathrm{C}$ \\
10 & 4 & $\mathrm{D}$ \\
12.5 & 5 & E or DD \\
15 & 6 & $\mathrm{~F}$ \\
17.5 & 7 & $\mathrm{G}$ \\
20 & 8 & $\mathrm{H}$ \\
\hline
\end{tabular}

Patients were followed up for a mean duration of 18 months (median 21 months, range 12 to 24 months), all procedures were prospectively analyzed. All patients gave their informed consent to participate in the study, informed consent was also given for clinical photographs to be used for academic purposes.

The primary end points were the local wound complication and explantation rates. Secondary end points included local recurrence, the impact of the reconstruction on patient quality of life, patient satisfaction with the esthetic outcome, and an objective assessment of the esthetic outcome. The quality of life was assessed using the Breast Q Questionnaire, a method of assessing patient reported outcomes to study the effectiveness and impact of breast surgery from the perspective of the patient. ${ }^{5}$ This was posted out to patients following their surgery and can be seen in Table 2 . The crude Breast Q score, which was out of 90 points, was calculated and converted into percentages. The results were then further classified as very satisfied $\geq 68$ points ( $\geq 75 \%)$, satisfied $45-67$ points $(50-75 \%)$, and dissatisfied $\leq 45$ points $(\leq 50 \%)$. Patients were also asked to complete a patient satisfaction questionnaire to assess the esthetic outcome of surgery subjectively, which was scored between 0 and 10, where 10 indicated an excellent esthetic outcome and 0 indicated a poor outcome. This assessment was based on the Harvard scale described by Harris et al. ${ }^{6}$
Table 2: Breast $\mathbf{Q}$ questionarre; V.Dis=Very dissatisfied, Dis=Dissatisfeid, Sat=satisfied, V.Sat=Very Satisfied

\begin{tabular}{|c|c|c|c|c|}
\hline Question & v.Dis & Dis & Sat. & v.Sat \\
\hline $\begin{array}{l}\text { How you look in the mirror } \\
\text { Clothed? }\end{array}$ & 1 & 2 & 3 & 4 \\
\hline $\begin{array}{l}\text { The shape of your } \\
\text { reconstructed breast when you } \\
\text { are wearing bra }\end{array}$ & 1 & 2 & 3 & 4 \\
\hline $\begin{array}{l}\text { How normal you feel in lyour } \\
\text { clothes }\end{array}$ & 1 & 2 & 3 & 4 \\
\hline $\begin{array}{l}\text { The size of your reconstructed } \\
\text { breasts }\end{array}$ & 1 & 2 & 3 & 4 \\
\hline $\begin{array}{l}\text { Being able to wear clothing } \\
\text { that is more fitted }\end{array}$ & 1 & 2 & 3 & 4 \\
\hline $\begin{array}{l}\text { How your breast are lined up } \\
\text { in relation to each other }\end{array}$ & 1 & 2 & 3 & 4 \\
\hline How comfortably your bras fit & 1 & 2 & 3 & 4 \\
\hline $\begin{array}{l}\text { The softness of your } \\
\text { reconstructed breast }\end{array}$ & 1 & 2 & 3 & 4 \\
\hline $\begin{array}{l}\text { How equal in size your breast } \\
\text { are to each other }\end{array}$ & 1 & 2 & 3 & 4 \\
\hline $\begin{array}{l}\text { How natural your reconstructed } \\
\text { breast looks }\end{array}$ & 1 & 2 & 3 & 4 \\
\hline $\begin{array}{l}\text { How natural your reconstructed } \\
\text { breast sits/hangs }\end{array}$ & 1 & 2 & 3 & 4 \\
\hline $\begin{array}{l}\text { How your reconstructed breast } \\
\text { feel to touch }\end{array}$ & 1 & 2 & 3 & 4 \\
\hline $\begin{array}{l}\text { How much your reconstructed } \\
\text { breasts feels like a natural part } \\
\text { of your body }\end{array}$ & 1 & 2 & 3 & 4 \\
\hline $\begin{array}{l}\text { How closely matched your } \\
\text { breasts are to each other }\end{array}$ & 1 & 2 & 3 & 4 \\
\hline $\begin{array}{l}\text { How you look in the mirror } \\
\text { unclothed }\end{array}$ & 1 & 2 & 3 & 4 \\
\hline
\end{tabular}

\section{Surgical techniques}

Preoperative marking was done while the patient was standing. In medium size breast Grissoti's technique was planned (Figure 1), a curvilinear flap was marked on the breast with a diameter equivalent to the diameter of the central quadrantectomy incision in its upper part and tapered downwards and laterally toward the infra mammary fold, the skin disc, which forms the new areola was drawn in the upper part of the flap of $4 \mathrm{~cm}$ diameter. 


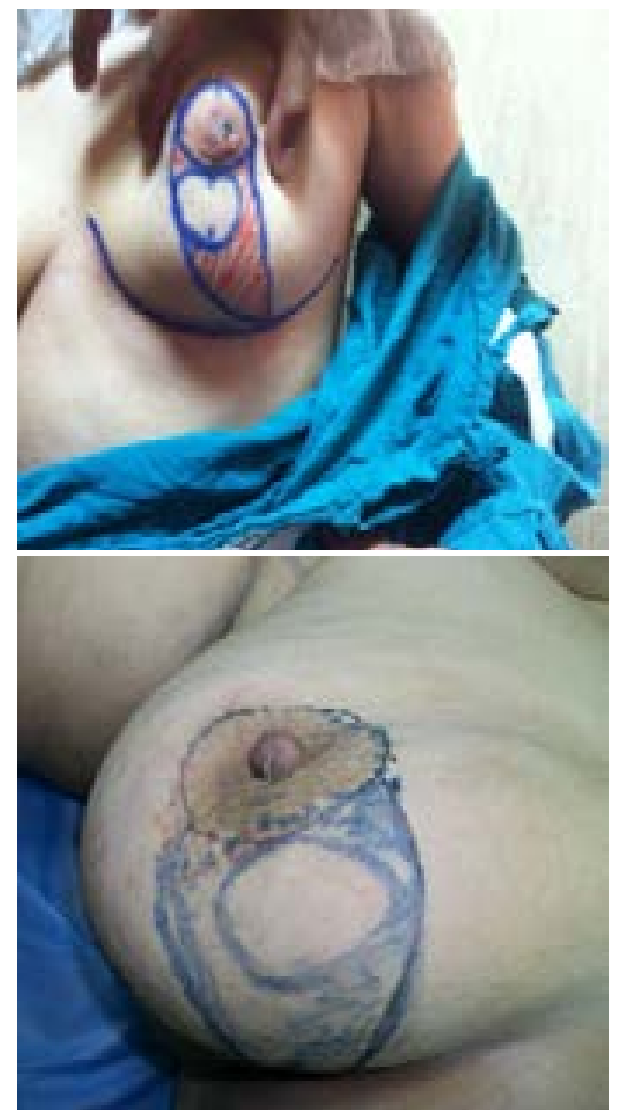

Fig 1: Preoperative marking of the incision for Grissoti's local skin-glandular flap.

In large size breast inferior pedicle technique was applied; (Figure 2) a key-hole excision, including the NAC, followed by an inferior advancement pedicle carrying skin island from the inferior pole of the breast on the upper half of the pedicle.

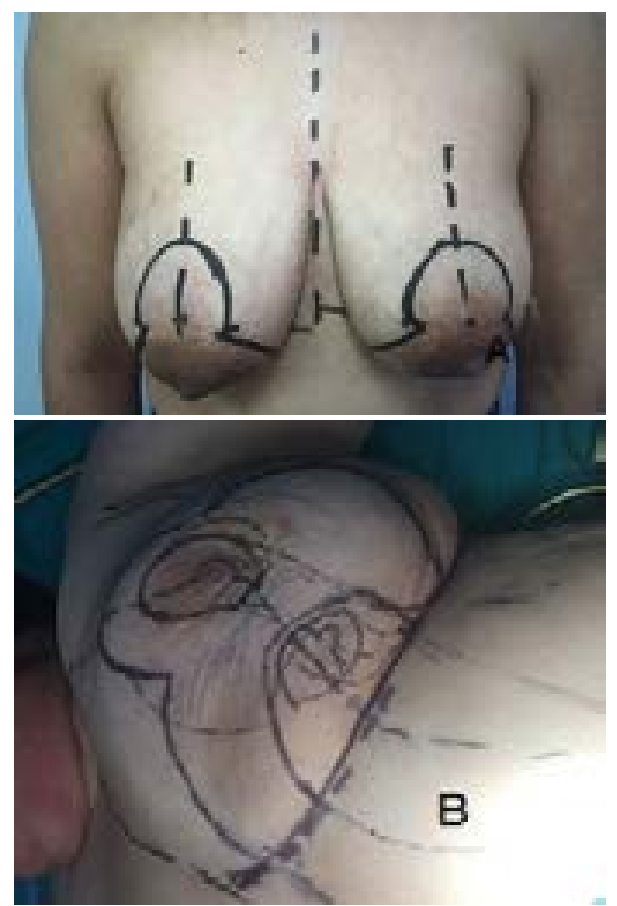

Fig 2: Preoperative marking of inf. Pedicle flap, A: while the patient is standing, $B$ : in supine position showing the inferior pedicle with skin island.
Under general anesthesia, the patient was placed in supine position with the ipsilateral arm abducted 90 degrees. Central quadrantectomy including excision of NAC down to the pectoralis fascia (Figure 3A), marking of tumor bed with clips was performed. The specimen was marked with one stitch at 12 O'clock and sent for immediate frozen histopathology examination for surgical margins. We insured an adequate safety margin of $1 \mathrm{~cm}$. Breast reconstruction was used Grissoti's technique for medium size breast "cup B-C" and skin island on inferior pedicle mammoplasty for large size breast "cup D or more".

In Grissoti's technique the flap was de-epithelialized carefully except for the skin disc "future areola" (Figure 3A). The medial edge of the flap was incised down to the pectoral fascia (Figure 3B), undermined for $4 \mathrm{~cm}$ to facilitate the supero-lateral rotation of the flap. The deep surface of the flap was sutured to the deep aspect of the defect with $3 / 0$ absorbable polyglactin sutures, then the circular incision was closed around the skin disc in two layers. A suction drain was placed on that stage, lying on the pectoral fascia. The medial and lateral pillars of the breast were closed together burying the de-epithelialized surface (Figure 3C).

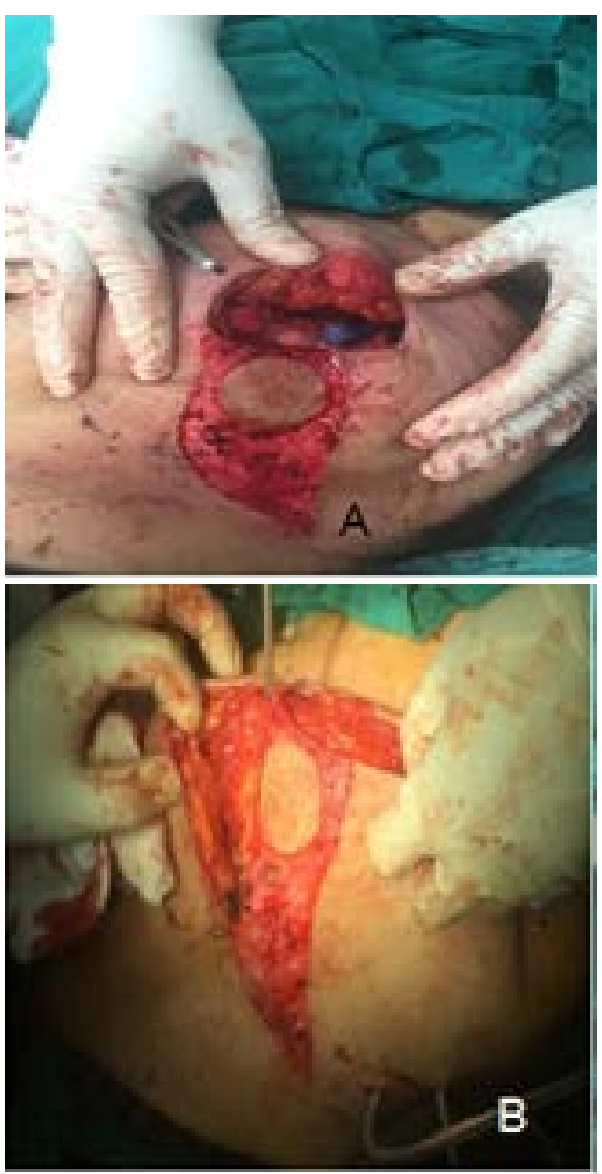




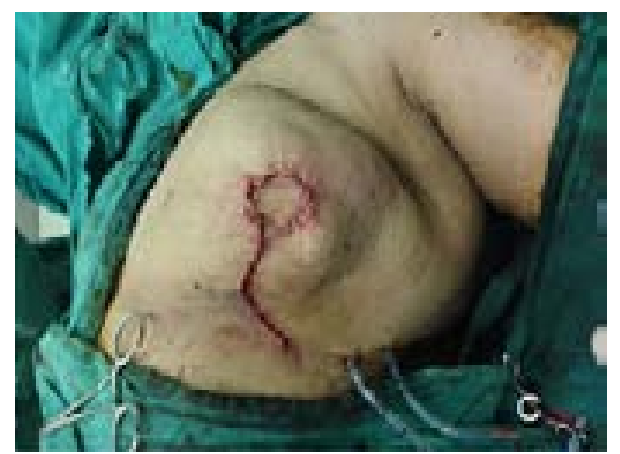

Fig 3: A-NAC is excised down to pectoralis fascia, de-epithelialized carefully except for the skin disc which forms the new areola, B- flap is undermined for $4 \mathrm{~cm}$, together with the medial and lateral pillars of the breast to facilitate the supero-medial rotation of the flap, $\mathrm{C}$ - medial and lateral pillars of the breast are closed together burying the de-epithelialized surface.

In inferior pedicle technique the skin was incised along the drawn markings and the inferior pedicle was de-epithelialized except for the skin disc 'future new areola" (Figure 4A). Skin flaps of 2 $\mathrm{cm}$ in thickness were dissected superiorly, medially and laterally down to the pectoralis fascia. Care was taken not to overextend the dissection to avoid compromising of blood supply. The inferior pedicle was prepared with a base of 6 to $12 \mathrm{~cm}$ in width and a thickness of 2 to $6 \mathrm{~cm}$ then transferred superiorly to fulfill the defect. The skin was closed temporarily with skin staples (Figure 4B). The final nipple position was drawn on the breast midline $6 \mathrm{~cm}$ from the inframammary fold (Figure 4C). The inferior pedicle was elevated through the incision with care to avoid folding or rotating the pedicle.
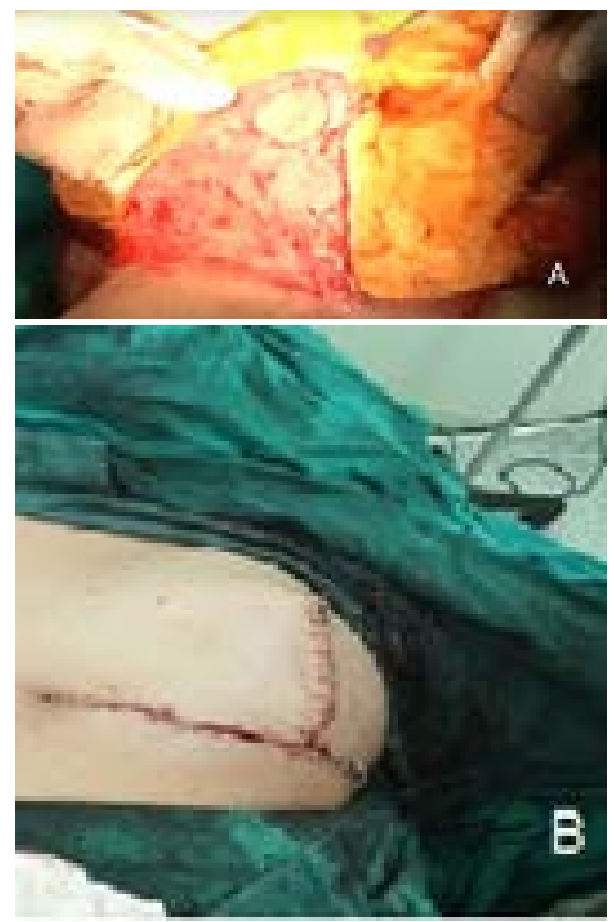

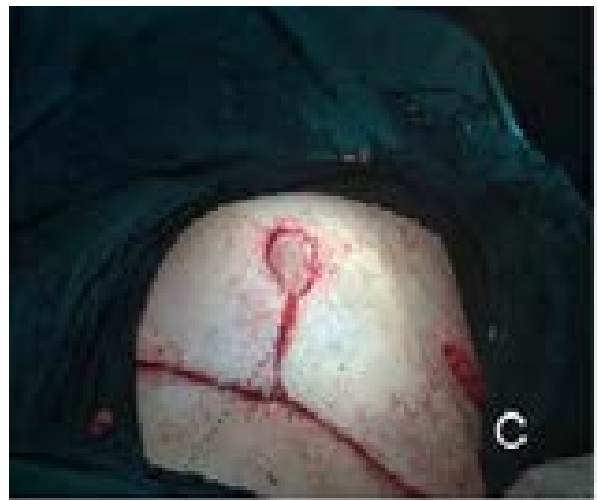

Fig 4A: The skin was incised along the drawn markings and the inferior pedicle was de-epithelialized except for the skin disc which forms the new areola just inferior to the areola, B: The skin is closed temporarily with skin staples and symmetry is assessed in the sitting position, C: The final nipple position is drawn on the breast midline $6 \mathrm{~cm}$ from the inframammary fold.

Finally, in both procedures axillary clearance of level 1 and 2 lymph node was done through a separate transverse axillary incision. At the end of the procedure the wounds were covered with light non-compressible dressings. For each operation, the surgical prophylaxis included Povidone Iodine solution skin preparation and $1 \mathrm{gm}$ ampicillinsulbactam was given intravenously 30 minutes before the surgical incision and every 12 hour along their hospital stay. Patients were maintained on oral $1 \mathrm{gm}$ of ampicillin-sulbactam for 7 days.

\section{Statistical Analysis}

Statistical evaluation was properly done using $T$ test, $x 2, S D, P$ value and Mass Whitney $t$.

\section{Result}

Thirty female patients with central breast tumors undergoing central quadrantectomy were identified. The mean age $45.4 \pm 13.2$ years with a range of 29 to 60 years. The mean BMI was 24.5. Twelve patients were pre-menopausal while, the rest eighteen patients were post-menopausal. Twenty-six patients were married. Twenty-five patients presented with a palpable mass, the mass was strictly retroareolar in 15 patients and in 10 patients the mass extended in the peri-areolar region to a maximum distance of $1.5 \mathrm{~cm}$ beyond the areolar margin.

Upon breast ultrasonography, the size of the tumors ranged from 6 to $31 \mathrm{~mm}$ with a mean diameter of $15.9 \pm 8.6 \mathrm{~mm}$, two patients with Paget's disease had no detectable mass, either clinically or mammography apart from the retro-areolar parenchymal distortion and clustered micro-calcification which was confirmed with MRI. 
All patients were staged according to the (TNM) classification system (Table 3). Final pathological result showed positive axillary nodes in $22 / 30$ patients.

Table 3: TNM classification of $\mathbf{3 0}$ patients with central breast cancer

\begin{tabular}{lc}
\hline $\begin{array}{l}\text { Preoperative stage } \\
\text { Tumor (T) }\end{array}$ & NO. of cases \\
\hline Tis & 2 \\
T1 & 12 \\
T2 & 16 \\
T3 & 0 \\
T4 & 0 \\
Lumph node (N) & \\
N0 & 11 \\
N1 & 19 \\
N2 & 0 \\
Metastasis (M) & \\
M0 & 30 \\
M1 & 0 \\
\hline
\end{tabular}

All final surgical margins were clear of residual disease. The two patients with Paget's showed low grade DCIS and free safety margins.

The operative time ranged from 90 to 150 minutes with a mean of $115 \pm 14.8$ minutes, No patient required intra or post-operative blood transfusion. Patients were hospitalized for a range of 1 to 3 days with a median of 2 days.

All patients were discharged with their drains and asked to come back for regular dressings and drain removal when indicated, breast drains were removed after 4-7 days (median: 5 days), while axillary drains were removed after 8-12 days (median: 10 days).

The primary end points were the local wound complication, one patient 3.33\% developed partial flap sloughing which was superficial and subsided with only dressings and two cases of breast seroma. No cases of full thickness flap sloughing, axillary seroma, hematoma or wound sepsis were seen. By statistical analysis, the incidence of early and late complications failed to reach statistical significance except for volume discrepancy (Table 4).

As for the secondary endpoints, over a mean followup of 18 months, we observed one case (3.33\%) of local recurrence in upper outer quadrant of the breast in Grissoti's group.

Of thirty patients, all questionnaires were returned. The mean percentage Breast Q score was 73 points $(81 \%)($ median $=87 \%$; range $=61.66 \%-100 \%)$. There was a high level of satisfaction with 24 patients (80\%) having a Breast Q score more than or equal to 67 points and $13.3 \%$ between 45 and 67 points $(50 \%-74 \%)$. Two patients $(6.6 \%)$ were dissatisfied. A high level of patient satisfaction was likewise established through a mean subjective score of esthetic outcome of 8.1 out of a possible 10 , with a median score of 10 (range $=6$ to 10$)$.

Table 4: Postoperative complications. Declare $\mathbf{P}$ larger than alpha $=0.05$ insignificant

\begin{tabular}{lccc}
\hline Postoperative complication & No. of cases & Percentage & P value \\
\hline Early before $\mathbf{6}$ weeks & 1 & & \\
Superficial flap sloughing & 0 & 3.33 & 0.30951 \\
Full thickness flap sloughing & 0 & 0 & \\
Wound seroma & 2 & 0 & 0.3489 \\
Haematoma & 0 & 6.66 & \\
Wound sepsis & 0 & 0 & \\
Axillary seroma & & 0 & 0.0005 \\
Late After $\mathbf{6}$ weeks & 18 & 58 & 0.30971 \\
Volume discrepancy & 1 & 3.33 & 0.34893 \\
Shape distortion & 2 & 6.66 & 0.30971 \\
Bad scarring & 2 & 3.33 & \\
Local recurrence & & & \\
\hline
\end{tabular}


All patients refused contra-lateral symmetralization mammoplasty at the time of operation, however five patients of the inferior pedicle group asked for contralateral symmetralization after completing their adjuvant treatment.

On the other hand, none of the patients agreed on NAC reconstruction. Adjuvant treatment was started in all cases without delay. Early postradiation breast edema occurred in all patients, but gradually settled after treatment completion.

\section{Discussion}

The main principle in the surgical management of breast cancer will remain the oncologic safety. Although, acceptable aesthetic results have an important impact on quality of the patient's life, improving self-image and self-confidence. For this breast surgeon should exhaust their selves to maximize cosmetic outcomes when surgically managing breast cancer.

In our study, both techniques results were in a superior cosmetic outcome with preservation of natural breast shape and appearance, providing psychological benefits to patient by avoiding feelings of mutilation and loss of femininity.

This study proved both techniques allowed easy access to central quadrantectomy ensuring safety margin with low wound complication rate of $3.33 \%$ despite the high incidence of chemotherapy and radiotherapy. We reported one case of partial flap necrosis at $T$ shape incision of inferior pedicle technique which was treated with local dressing without surgical intervention. Our explanation was increased tension on skin flaps due to an extensive reduction in that huge breast and 2 cases of breast seroma, our results were nearly similar to Farouk et al. ${ }^{7}$ Other factors which have also been associated with increased necrotic complication rates include higher BMI, large breast size, and smoking. ${ }^{8}$ Our patient cohort had a relatively low BMI of 24.5 which may have led to more favorable outcomes. Therefore, with very few reported complications in our series, both approaches would be the ideal procedure with low complication rates in selected, low-risk patients.

In the past, central tumors, which represent $5-20 \%$ of breast cancers have been considered to be more serious, multifocal and more likely to recur. In fact, it has been demonstrated that central quadrentectomy does not compromise the oncological safety in selected patients. ${ }^{9}$

Nipple infiltration was found in $60 \%$ of cases confirming the importance of NAC excision in central tumor even without the clinical involvement of nipple that was higher than other studies ranging
$35-58 \%,{ }^{10}$ mostly due to more T2 tumors in our study "more advanced cases than these studies".

We had only one case $3.33 \%$ of local recurrence in Grissoti's group which is nearly similar to local recurrence in breast conserving surgery for any breast quadrant as shown to be $8-10 \%{ }^{11}$ and $1-3 \%$ in another study. ${ }^{12}$ Overall survival, risk of recurrence and metastatic disease are identical to other tumor locations. ${ }^{9}$

Overall, patients in our case series were satisfied with the results of their reconstructive surgery. The high Breast Q score indicates that the use of these techniques lead to a measurable improvement in the quality of life, making it an excellent choice in selected patient cohorts. Similar findings were established in various other studies. Fitzal ${ }^{13}$ showed that women undergoing central quadrantectomy with reconstruction felt more comfortable with their body image as compared with those performing mastectomies, with a significantly lower sense of mutilation. Such women reported a better coping with the traumatic experience of breast cancer and losing a breast. In addition, the high-patient satisfaction scores demonstrated in our study indicate that the majority of patients were pleased with the outcome of their reconstructive surgery. This was further validated by the high score in the objective assessment demonstrating an excellent cosmetic outcome.

In our study five patients sought for contralateral symmetrization after completing their adjuvant therapy all of them in the inferior pedicle group, confirming that this technique reduced the volume of the breast significantly; two of these patients reported poor aesthetic result for the procedure regarding the discrepancy of breast volume. However, all the patients were informed about the expected discrepancy of breast volume after the operation none of them agreed for synchronous contralateral symmetrization. Our findings are consistent with Naguib ${ }^{14}$ who demonstrated that all patients refused to undergo contra-lateral mammaplasty for symmetrization. When this option was offered synchronously with tumor ablation, patients feared bilateral scarring and disfigurement.

No patient agreed for NAC reconstruction, it appears that simple preservation of the breast mound for a feminine body contour is an essential requirement for women, especially in a conservative society like Egypt. This is similar to what has been reported by Moustafa A and Fakhr 2014. ${ }^{15}$

This study was not without its limitations. First, we report on a relatively small patient cohort. Second, the follow-up period is relatively short at just 18 
months. There is a need in the future for studies with larger patient cohorts being followed up over longer periods of time in order to fully evaluate potential long-term complications. Finally, the questionnaires sent were not blinded which may have weakened the objectivity of the reported excellent esthetic outcomes. Nonetheless, our findings do demonstrate the safety and feasibility of both approaches.

\section{Conclusion}

For years central and retro-areolar breast cancer were treated by mastectomy either alone or combined with reconstruction. Now, the two procedures used in the present study have undoubtful cosmetic and functional advantages and fulfill the oncological safety without mastectomy.

This is in addition to simplicity and a shorter recovery time. It is ideal for women with medium to large breasts, having a Paget's disease or harboring central tumors that do not exceed $1.5 \mathrm{~cm}$ from the areola. The main disadvantage of this technique is the loss of NAC. Nevertheless, it can be easily restored by tattooing and minimal surgery if needed, Another drawback of the procedure is the need for contra-lateral symmetrization when volume discrepancies between both breasts are too significant especially in the inferior pedicle technique.

\section{References}

1. Ibrahim A, Khaled $H$, Mikhail $N$, Kamel $H$, Baraka H: Cancer incidence in Egypt: Results of the National Population-Based Cancer Registry Program. Journal of Cancer Epidemiology 2014; Article ID 437971, 215-232.

2. Onitilo A, Engel $M$, Stankowski V, Doi R: Survival comparisons for breast conserving surgery and mastectomy revisited: Community experience and the role of radiation therapy. Clinical Medicine \& Research 2015; 13: 65-73.

3. Skillman JM, Humzah MD, Brown IM, et al: The future of breast surgery: A new subspecialty of oncoplastic breast surgeons. Breast 2003; 12: 161-162.

4. Schoeller $T$, Huemer GM: Immediate reconstruction of the nipple/ areola complex in oncoplastic surgery after central quadrantectomy. Ann Plast Surg 2006; 57: 611-615.

5. Pusic AL, Klassen AF, Scott AM: Development of a new patient reported outcome measure for breast surgery: The BREAST-Q. Plast
Reconstr Surg 2009; 124: 345-353.

6. Harris JR, Levene MB, Svensson G, et al: Analysis of cosmetic results following primary radiation therapy for stages I and II carcinoma of the breast. Int J Radiat Oncol Biol Phys 1979; 5: 257-261.

7. Farouk O, Attia E, Roshdy S, Khater A, Senbe A, Fathi A, Denewer A: The outcome of oncoplastic techniques in defect reconstruction after resection of central breast tumors. World Journal of Surgical Oncology 2015; 13: 285286.

8. Algaithy ZK, Petit JY, Lohsiriwat V: Nipple sparing mastectomy: Can we predict the factors predisposing to necrosis? Eur J Surg 2012; 38: 125-129.

9. Fitoussi A, Berry, MG, Couturaud B, Salmon $R$ : Oncoplastic and reconstructive surgery for breast cancer. Berlin: Springer-Verlag Heidelberg. $2^{\text {nd }}$ edn. 2009.

10. Galimberti V, Zurrida S, Zanini V, Callegari M, Veronesi P, Catania $S$, et al: Central small size breast cancer: How to overcome the problem of nipple and areola involvement. Eur J Cancer 1993; 29A: 1093-1096.

11. Fisher B, Anderson S, Bryant J: Twenty-year follow-up of a randomized trial comparing total mastectomy, lumpectomy, and lumpectomy plus irradiation for the treatment of invasive breast cancer. Engl J Med 2002; 347: 12331241.

12. Arvold ND, Taghian AG, Niemierko A: Age, breast cancer subtype approximation, and local recurrence after breast-conserving therapy. J Clin Oncol 2011; 29: 3885-3891.

13. Fitzal F.: An oncoplastic procedure for central and mediocranial breast cancer. Eur J Surg Oncol 2007; 38: 1016-1019.

14. Sherif N: Oncoplastic resection of retroareolar breast cancer: Central quadrantectomy and reconstruction by local skin-glandular flap. Journal of the Egyptian Nat Cancer Inst 2006; 18: 334-347.

15. Moustafa A, Fakhr I: Outcome of different oncoplastic surgical (OPs) techniques for centrally located breast cancer (CLBC). Journal of the Egyptian National Cancer Institute 2014; 26: 203-209. 